推论 1 Levi 方程 $u_{t}=\left(\begin{array}{l}q \\ r\end{array}\right)_{t}=X_{m}$ 的 充分必要条件是

$$
L_{t}=\left[W_{m}, L\right](m=0,1 ; \cdots) .
$$

推论 2 位势函数 $u(x)=\left(\begin{array}{l}q(x) \\ r(x)\end{array}\right)$ 满足 定态的 Levi 系统: $X_{N}+a_{1} X_{N-1}+\cdots+$ $a_{N} X_{0}=0$ 的充要条件是

$$
\left[W_{N}+a_{1} W_{N-1}+\cdots+a_{N} W_{0}, L\right]=0,
$$

$a_{k}(k=1, \cdots N)$ 为常数.

致谢：感谢曹策问教授对本文的指点.

\section{参考 文 献}

[1] 曹策问,科学通报, 34(1989), 10:723-724.

[2] Lax, P.D., SIAM Rev., 18(1976), 351--375. 乔志军 (辽宁大学数学系, 沈阳 110036)

\title{
图与补图的 $n$-全色数*
}

定义 1 对图 $G(V, E)$ 和自然数 $n$, 对 其长度不大于 $n$ 的路上所有点(或所有边、或 所有点和所有边)均染为不同色, 其所用颜色 的最少数目称为 $G$ 的 $n$-色数 (或 $n$-边色数、 或 $n$-全色数)，简记作 $\chi_{n}(G)$ （或 $\chi_{n}^{\prime}(G)$ 、 或 $\left.\chi_{n}^{T}(G)\right)$.

定义 2 对图 $G(V, E)$ 和自 然数 $n$, $D_{n} \subseteq V$ (或 $D_{n}^{\prime} \subseteq E$ 、或 $D_{n}^{T} \subseteq V \cup E$ ), $D_{n}$ (或 $D_{n}^{\prime}$ 、或 $D_{n}^{T}$ ) 中任意两点（或任意两边、或任 意两个元素)均在 $G$ 的某一条长不大于 $n$ 的 路上, 则称 $D_{n}$ (或 $D_{n}^{\prime}$, 或 $D_{n}^{T}$ ) 为 $G$ 的 $n$-完 全集(或 $n$-边完全集、或 $n$-全完全集).

引理 $1 \chi_{n}^{T}(G) \geqslant \max \left\{\left|D_{*}^{T}\right| \mid D_{*}^{T}\right.$ 为 $G$ 的 $n$-全完全集 $\}$.

引理 $2 \chi_{4}^{\prime}(G) \geqslant\left[\frac{(2 q+p) q}{p^{2}}\right]$, 且界不 可改进, 其中 $p=|V|, q=|E|,\lceil x\rceil$ 为 不小于 $x$ 的最小整数.

引理3 $\chi_{4}^{\tau}(G) \geqslant\left[\frac{(2 q+p) q}{p^{2}}\right]+\frac{2 q}{p}+1$, 且界不可改进。
定理 1 记 $\bar{G}$ 为 $G$ 的补图, 则

$$
\begin{gathered}
{\left[p^{2}+4 p+3\right] \leqslant \chi_{4}^{T}(G)+\chi_{4}^{T}(\bar{G})} \\
\leqslant \frac{p(p+3)}{2} .
\end{gathered}
$$

定理 2 对 $n \geqslant 5$, 有

$$
\begin{aligned}
\left\lceil\frac{3 p^{2}+10 p-1}{8}\right] & \leqslant \chi_{n}^{T}(G)+\chi_{n}^{T}(\bar{G}) \\
& \leqslant \frac{p(p+3)}{2},
\end{aligned}
$$

且上界不可改进, 下界当 $p$ 为偶数时不可改 进。

问题 1 确 定 $n=3 、 4$ 时, $\chi_{n}^{\mathrm{T}}(G)+$ $\chi_{*}^{T}(\bar{G})$ 的可达下界.

张忠辅

（兰州铁道学院，兰州 730070）

孙良

(北京理工大学,北京 100081) 目.

\footnotetext{
* 国家自然科学基金和甘肃省自然科学基 金资 助项
}

\section{Square-full 整数的分布}

设 $\varepsilon>0$. 令 $A(x)$ 表示 $\leqslant x$ 的Square- full 整数之个数. 


$$
A(x)=\frac{\zeta\left(\frac{3}{2}\right)}{\zeta(3)} x^{\frac{1}{2}}+\frac{\zeta\left(\frac{2}{3}\right)}{\zeta(2)} x^{\frac{1}{3}}+\Delta(x),
$$

这里 $\Delta(x)$ 称为 $A(x)$ 的余项.

文献[1]首先证得: $\Delta(x)=O\left(x^{\frac{13}{81}+e}\right)$. 1988 年, Ranachadra 等又得到了

$$
\Delta(x)=O\left(x^{\frac{11}{72}+8}\right) \text {. }
$$

定理 1 在 Riemann 假设下,对任意的 $\boldsymbol{Y} \geqslant 1$,

$$
\begin{aligned}
\Delta(x)= & -\sum_{\delta<Y} \mu(\delta) \sum_{n^{5} \delta^{6<x}} \\
& \times\left\{\psi\left(\left(\frac{x}{\delta^{6} n^{3}}\right)^{\frac{1}{2}}\right)\right. \\
& \left.+\psi\left(\left(\frac{x}{\delta^{6} n^{2}}\right)^{\frac{1}{3}}\right)\right\}
\end{aligned}
$$

$$
+O\left(x^{\frac{1}{6}+\frac{8}{2}} Y^{-1+\frac{8}{2}}+Y\right) \text {, }
$$

其中 $\psi(\theta)=\theta-[\theta]-\frac{1}{2}$.

推论 ${ }^{[2]}$ 在 Riemann 假设下, 有

$$
\Delta(x)=O\left(x^{\frac{11}{12}+8}\right) \text {. }
$$

定理 2 若 Riemann 假设成立, 对任意 的 $\varepsilon>0$,

$$
\Delta(x)=O\left(x^{\frac{5}{33}+8}\right) .
$$

\section{参文献} [1] Suryanarayana, D. and Sitara machandra, R.,
Arkiv Mat., B II(1973), 2: 195-201.

曹晓东

（北京市 10061 信箱,北京 102549）

\section{负 Ricci 曲率紧 Riemann 流形上第一特征值的估计}

本文在 $n$ 维紧 Riemann 流形 $M$ 上考虑 下述问题: $-\Delta u-\lambda u$, 得到了关于具负 下界 Ricci 曲率的流形的第一非零 特 征 值 $\lambda_{1}$ 的两种互不包含的估计, 改进了文献[ 1 的 主要结果.

本文首先得到第一特征函数的梯度估 计. 设 $u$ 满足

$$
\left\{\begin{array}{l}
-\Delta u=\lambda_{1} u ; \\
\max u=1-\delta, \\
\min u=-k(1-\delta),
\end{array}\right.
$$

其中 $0<k \leqslant 1, \delta>0, \delta$ 是充分小的任意 常数.

令

$$
\begin{aligned}
& f=\frac{u-\frac{1-k}{2}(1-\delta)}{(1+k) / 2}, \\
& a-\frac{1-k}{1+k}, \\
& \varphi(x)=\arcsin f(x),
\end{aligned}
$$

$$
F\left(\varphi_{0}\right)=\sup _{x \in M, \varphi(x)=\varphi_{0}}|\nabla \varphi(x)|^{2} .
$$

引理 A

$$
F(\varphi) \leqslant\left\{\begin{array}{c}
{\left[\sqrt{(1+a) \lambda_{1}}+\alpha \sqrt{L} \cos \varphi\right]^{2},} \\
\varphi \in\left[0, \frac{\pi}{2}-\delta_{1}\right], \\
\left(\sqrt{\lambda_{1}}+\alpha \sqrt{L} \cos \varphi\right)^{2}, \\
\varphi \in\left[-\frac{\pi}{2}+\delta_{1}, 0\right],
\end{array}\right.
$$

其中

$$
\begin{aligned}
& \alpha=\frac{1}{2} \max \{\sqrt{n-1}, \sqrt{2}\}, \\
& \delta_{1}=\arcsin \sqrt{\delta(2-\delta)} .
\end{aligned}
$$

\section{引理 B}

$$
F(\varphi) \leqslant\left\{\begin{array}{c}
\lambda_{1}+L g(\varphi)+\lambda_{1} a H(\varphi), \\
\varphi \in\left[-\frac{\pi}{2}+\delta_{1}, 0\right], \\
\lambda_{1}+L+\lambda_{1} a H(\varphi), \\
\varphi \in\left[0, \frac{\pi}{2}-\delta_{1}\right],
\end{array}\right.
$$

